\title{
A New Highly Reactive and Low Lipophilicity Fluorine-18 Labeled Tetrazine Derivative for Pretargeted PET Imaging
}

\author{
Outi Keinänen, ${ }^{\dagger}$ Xiang-Guo Li, ${ }^{\ddagger}$ Naveen K. Chenna, ${ }^{\S}$ Dave Lumen, ${ }^{\dagger}$ Jennifer Ott, ${ }^{\dagger}$ Carla F. M. Molthoff, ${ }^{\perp}$
} Mirkka Sarparanta, ${ }^{\dagger} \#$ Kerttuli Helariutta, ${ }^{\dagger}$ Tapani Vuorinen, ${ }^{\S}$ Albert D. Windhorst, ${ }^{\perp}$ and Anu J. Airaksinen*, $\dagger$

${ }^{\dagger}$ Laboratory of Radiochemistry, Department of Chemistry, University of Helsinki, FI-00014 Helsinki, Finland

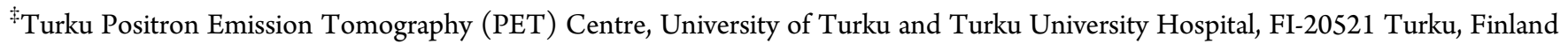

${ }^{\S}$ Department of Forest Products Technology, Aalto University School of Chemical Technology, FI-00076 Espoo, Finland

${ }^{\perp}$ Department of Radiology and Nuclear Medicine, VU University Medical Center, $1081 \mathrm{HV}$ Amsterdam, The Netherlands

\# Department of Radiology, Memorial Sloan Kettering Cancer Center, New York, New York 10065, United States

\section{Supporting Information}

ABSTRACT: A new ${ }^{18}$ F-labeled tetrazine derivative was developed aiming at optimal radiochemistry, fast reaction kinetics in inverse electron-demand Diels-Alder cycloaddition (IEDDA), and favorable pharmacokinetics for in vivo bioorthogonal chemistry. The radiolabeling of the tetrazine was achieved in high yield, purity, and specific activity under mild reaction conditions via conjugation with $5-\left[{ }^{18} \mathrm{~F}\right]$ fluoro-5deoxyribose, providing a glycosylated tetrazine derivative with low lipophilicity. The ${ }^{18}$ F-tetrazine showed fast reaction kinetics toward the

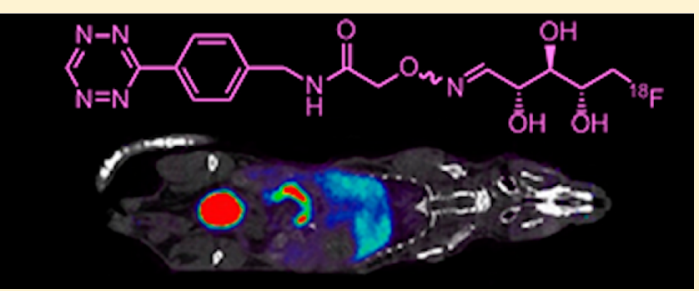
most commonly used dienophiles in IEDDA reactions. It exhibited excellent chemical and enzymatic stability in mouse plasma and in phosphate-buffered saline $(\mathrm{pH} 7.41)$. Biodistribution in mice revealed favorable pharmacokinetics with major elimination via urinary excretion. The results indicate that the glycosylated ${ }^{18} \mathrm{~F}$-labeled tetrazine is an excellent candidate for in vivo bioorthogonal chemistry applications in pretargeted PET imaging approaches.

KEYWORDS: Bioorthogonal chemistry, click chemistry, kinetics, PET imaging, tetrazine

$\mathrm{M}$ olecular imaging techniques play an increasingly important role in the clinic and in drug discovery and development. ${ }^{1,2}$ Positron emission tomography (PET) is a quantitative and sensitive imaging method that uses radiotracers labeled with positron-emitting radionuclides, allowing the detection of as little as picomoles of the radiotracer. ${ }^{3}$ Fluorine-18 $\left({ }^{18} \mathrm{~F}\right)$ is the most widely used PET isotope in nuclear medicine with half-life of $109.8 \mathrm{~min}$. The advantages of ${ }^{18} \mathrm{~F}$ include the ease of production with high yield and high specific activity. Furthermore, it has a low positron energy, which contributes to improved resolution and high quality of the PET image. ${ }^{4,5}$

Bioorthogonal chemistry has demonstrated great potential in molecular imaging. ${ }^{6-14}$ Tetrazine ligation with dienophiles exhibits the fastest reaction kinetics known in bioorthogonal chemistry so far, which is a great advantage for imaging under the tracer conditions of PET. ${ }^{15,16}$ The fastest kinetics with this inverse electron-demand Diels-Alder cycloaddition (IEDDA) of tetrazines are achieved with trans-cyclooctene (TCO). However, even simpler dienophile scaffolds such as norbornene show superior second order rate constants when compared to other bioorthogonal reactions. ${ }^{17}$ This makes the IEDDA reaction a lucrative candidate for the use of tracers labeled with short-lived isotopes for in vivo imaging. Recently, ${ }^{64} \mathrm{Cu}$ and ${ }^{111}$ In labeled tetrazines have been successfully used in pretargeted in vivo imaging of tumor specific monoclonal antibodies. $^{13,14,18}$ Despite the recent great progress in the synthetic development of new IEDDA ligands for PET imaging, no successful ${ }^{18} \mathrm{~F}$-labeled IEDDA ligands for pretargeted imaging in vivo have been reported so far.

The tetrazine ligation with TCO can be carried out in mild conditions with high radiochemical yields even with a shortlived isotope like ${ }^{11} \mathrm{C}$ (half-life $\left.=20.4 \mathrm{~min}\right) .{ }^{19} \mathrm{~A}{ }^{18}$ F-labeled TCO derivative has been successfully used as a prosthetic group in radiolabeling of peptides and proteins. ${ }^{9}$ The use of TCO as a radiolabeled tracer in vivo has failed due to rapid isomerization of TCO in plasma to its less reactive isomer, cis-cyclooctene (CCO), by copper-containing biomolecules. ${ }^{20}$ Therefore, the radiolabeling of TCO to accomplish in vivo bioorthogonal reaction has proven to be an unsuccessful strategy. Instead, radiolabeled tetrazine derivatives have been shown to maintain their reactivity also in vivo. ${ }^{12,14}$ When clinical applications of pretargeted PET imaging are considered, ${ }^{18} \mathrm{~F}$ would be the ideal isotope due to its general availability and optimal physical properties. $9,21,22$ However, due to the instability of the tetrazine

Received: August 12, 2015

Accepted: December 10, 2015

Published: December 10, 2015 
Scheme 1. Synthesis of $\left[{ }^{18} \mathrm{~F}\right] 1$ and $1^{a}$

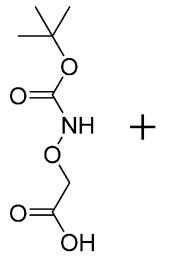

2

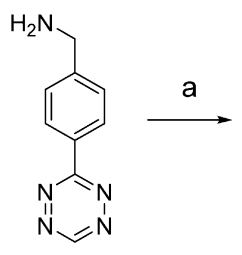

3

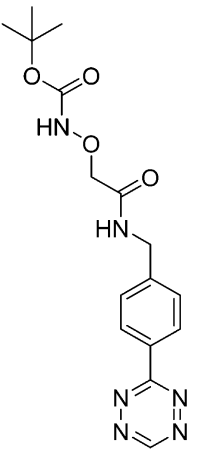

4

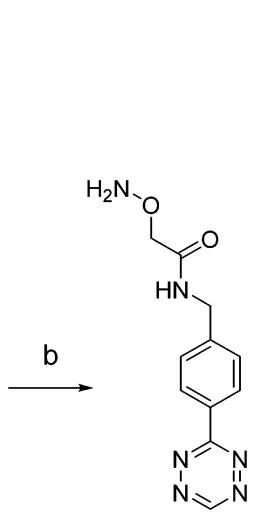

5

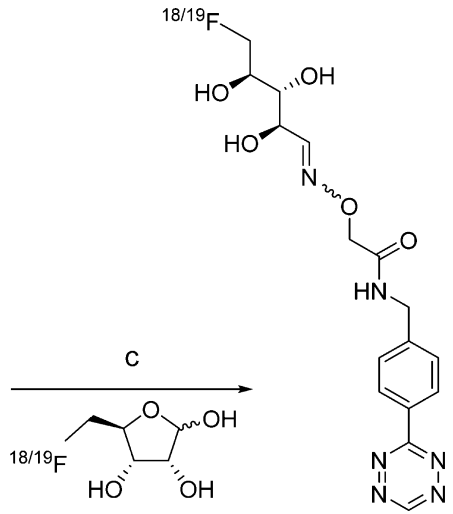

$\left[{ }^{18} \mathrm{~F}\right] 6,6$

$\left[{ }^{18} \mathrm{~F}\right] 1,1$

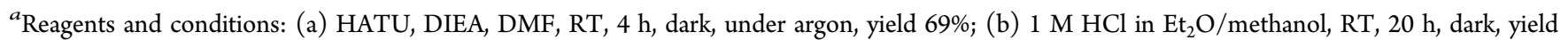
97\%; (c) $0.3 \mathrm{M}$ anilinium acetate buffer $\mathrm{pH} 4.6$, RT, $10 \mathrm{~min}$, yield $96 \%$, ratio of $E$ to $Z$ was $3: 1$.

moiety under conditions typically used in direct ${ }^{18} \mathrm{~F}$ fluorination, no ${ }^{18} \mathrm{~F}$-tetrazine was synthesized until very recently. ${ }^{23-25}$ A recently reported ${ }^{18} \mathrm{~F}$ propyl tetrazine was synthesized via a direct ${ }^{18} \mathrm{~F}$-fluorination only in low radiochemical yields (4-18\% decay corrected yield), but favorable pharmacokinetics in vivo. ${ }^{23}$ Successful and more efficient synthesis of ${ }^{18} \mathrm{~F}$-labeled tetrazine derivative was very recently published by using a $\left[{ }^{18} \mathrm{~F}\right] \mathrm{SiFA}-\mathrm{OH}$ building block, but influence of the relatively lipophilic alkylated organosilicon moiety on pharmacokinetics of the compound was not reported. ${ }^{24}$

Herein, we report an efficient synthetic method for preparation of a novel ${ }^{18} \mathrm{~F}$-labeled glycosylated tetrazine $\mathbf{1}$ (Scheme 1). The ${ }^{18}$ F-fluorination was achieved via oxime ether formation between 5 - $\left[{ }^{18} \mathrm{~F}\right]$ fluoro-5-deoxyribose $\left(\left[{ }^{18} \mathrm{~F}\right] 6\right)$ and aminooxy functionalized tetrazine $5 .\left[{ }^{18} \mathrm{~F}\right] 6$ can be synthesized in high yield and specific activity in less than $1 \mathrm{~h}^{26,27}$ The reaction of $\left[{ }^{18} \mathrm{~F}\right] 6$ with an aminooxy functional group is very fast at room temperature under mild reaction conditions in the presence of an aniline catalyst. We also hypothesized that $\left[{ }^{18} \mathrm{~F}\right]$ 1 would be more water-soluble compared to other radiolabeled tetrazines because of the three hydroxyl groups in the 5-fluoro5-deoxyribose moiety. Low lipophilicity is desired to achieve minimized nonspecific binding of the synthesized tetrazine in vivo. ${ }^{28-31}$ High lipophilicity also typically leads to higher levels of liver and intestinal radioactivity, making quantification of nearby organ uptake challenging. ${ }^{32,33}$ A low-lipophilicity, fastclearing tetrazine would be especially beneficial for pretargeted immunoimaging, resulting in substantially decreased radiation burden to nontarget tissues in contrast to using a conventionally radiolabeled antibody construct. ${ }^{34}$ In general, tracers with low lipophilicity have higher tendency to stay in the extracellular space unless actively transported. Fluorination at the five position is aimed to prevent phosphorylation of the 5$\left[{ }^{18} \mathrm{~F}\right]$ fluoro-5-deoxyribose $\left(\left[{ }^{18} \mathrm{~F}\right] 6\right)$ by ribokinase in hepatocytes, thus minimizing the possible nondesired liver accumulation due to active transportation and phosphorylation of ribose in the cells. ${ }^{35}$

Compound 4 was prepared in two steps from the BOCprotected aminooxy acetic acid (2) and 4-tetrazinylphenyl methamine (3), followed by deprotection of the aminooxy group by $\mathrm{HCl}$ (Scheme 1). The synthesized aminooxyfunctionalized tetrazine (5) was subsequently used for reaction with 5-fluoro-5-deoxyribose (6) to yield the fluorinated conjugate (1) in almost quantitative $96 \%$ yield. $\left[{ }^{18} \mathrm{~F}\right] \mathbf{1}$ was synthesized by using noncarrier added $\left[{ }^{18} \mathrm{~F}\right] 6$ with total overall $50.5 \pm 1.7 \%$ decay-corrected yield (starting from ${ }^{18} \mathrm{~F}^{-}$, mean \pm s.d., $n=5,317-1478 \mathrm{MBq}$ at the end of synthesis). The total duration of the synthesis was $2 \mathrm{~h}$. Radiochemical purity was $>99 \%$ and the specific activity up to $809 \mathrm{GBq} / \mu \mathrm{mol}$.

Compound $\mathbf{1}$ has an imine double bond and was found to exist as $E / Z$ isomers $(E: Z=3: 1)$. Isolated single $E$ and $Z$ isomers interconvert back into the original isomeric mixture over time (Figure 1).

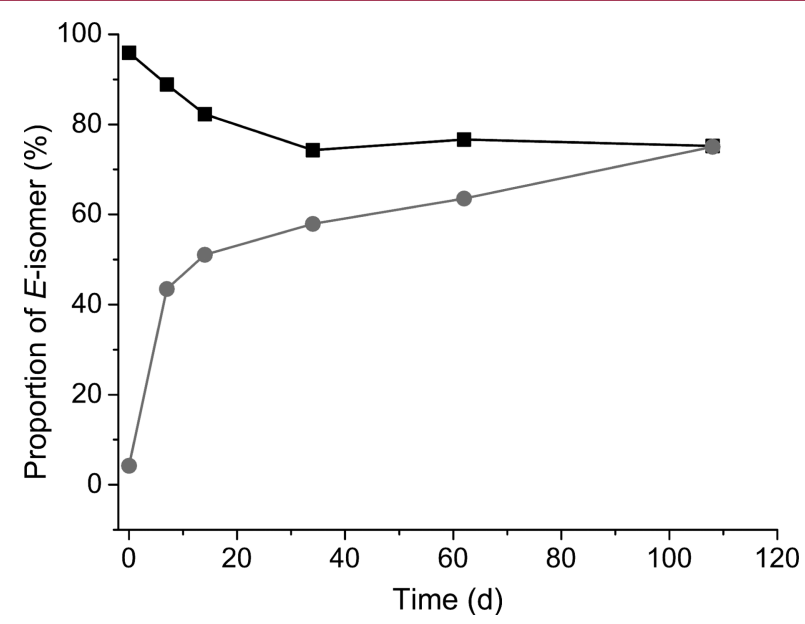

Figure 1. Isolated over $95 \%$ pure $E$ (black) and $Z$ (gray) isomers interconvert back into the original isomeric mixture $(75 \% \mathrm{E})$ over time.

In vitro stability of $\left[{ }^{18} \mathrm{~F}\right] \mathbf{1}$ in $\mathrm{PBS}(\mathrm{pH} 7.41)$ at room temperature $(\mathrm{RT})$ and in mouse plasma ( $50 \%$ whole plasma in PBS) at $37{ }^{\circ} \mathrm{C}$ was monitored over a period of $6 \mathrm{~h}$. At the designated time points samples were taken and injected to HPLC to determine the amount of intact $\left[{ }^{18} \mathrm{~F}\right] \mathbf{1}$. The isomers showed no difference in their stability (see SI, separate stability curves for $90 \% E$ and $90 \% Z$ isomers). Almost $90 \%$ of the intact $\left[{ }^{18} \mathrm{~F}\right] \mathbf{1}$ was observed after $2 \mathrm{~h}$ incubation in plasma, followed by relatively fast degradation reaching 50\% after $6 \mathrm{~h}$. The combined results of both isomers are presented in Figure 2. 


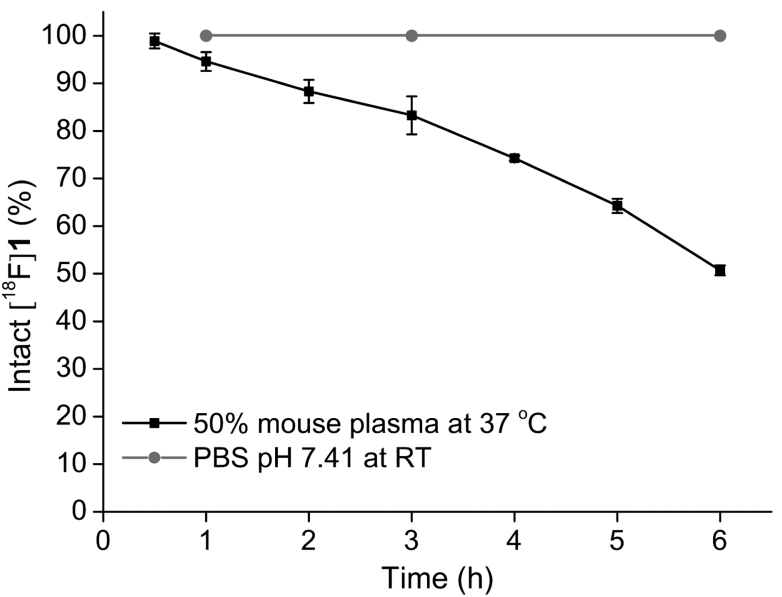

Figure 2. In vitro stability of $\left[{ }^{18} \mathrm{~F}\right] \mathbf{1}$ in $\mathrm{PBS} \mathrm{pH} 7.41(n=4)$ and $50 \%$ mouse plasma in PBS pH 7.41 ( $n=4$ until $4 \mathrm{~h}$, after that $n=2)$ over a period of $6 \mathrm{~h}$. No degradation products were observed in PBS. Values represent mean \pm s.d.

Lipophilicity of $\mathbf{1}$ was determined with the shake flask method by measuring the distribution coefficient of $\left[{ }^{18} \mathrm{~F}\right] \mathbf{1}$ between 1-octanol and $0.02 \mathrm{M}$ phosphate buffer at $\mathrm{pH} 7.41$. Interestingly, the isomers exhibited a slight difference in their lipophilicity. The $\log \mathrm{D}_{7.41}$ values for $E$ and $Z$ isomers were $-0.43 \pm 0.02$ and $-0.02 \pm 0.02(n=4$, mean \pm s.d. $)$, respectively.

Reaction rates of $\mathbf{1}$ were determined with three different dienophiles (Table 1). The reaction kinetics were measured

Table 1. Second Order Reaction Rate Constants $\left(k_{2}\right)$ for Reactions between 1 and Different Dienophiles ${ }^{a}$

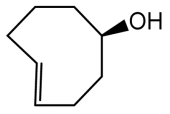

7

\begin{tabular}{clc} 
Dienophile & \multicolumn{1}{c}{ solvent } & $T\left({ }^{\circ} \mathrm{C}\right)$ \\
7 & plasma/PBS $1: 1^{b}$ & 37 \\
7 & methanol & 25 \\
8 & methanol & 25 \\
9 & methanol & 25
\end{tabular}

${ }^{a_{T}}$ The $k_{2}$ values are presented as mean \pm s.d. ${ }^{b} 50 \%$ mouse plasma in PBS, pH 7.41.

with a stopped flow spectrophotometer by monitoring characteristic tetrazine absorption at 270 or $534 \mathrm{~nm}$. All kinetic measurements were conducted under pseudo-first-order conditions in triplicate. The measurements were performed in 1020 -fold excess of dienophile in methanol, and for 7 also in mouse plasma (50\% in PBS, $\mathrm{pH} 7.41$ ). Reactions in methanol and plasma were monitored at 25 and $37{ }^{\circ} \mathrm{C}$, respectively. All kinetic measurements were performed with 1 in $E / Z$ forms ratio of 3:1. The second-order rate constants with different dienophiles are summarized in Table 1. As expected, significantly increased reaction rate was observed with 7 in mouse plasma compared to methanol. This is due to the hydrophobic effect that has been reported to increase IEDDA reaction rates. ${ }^{36}$

The ex vivo biodistribution of $\left[{ }^{18} \mathrm{~F}\right] \mathbf{1}$ was determined in male $\mathrm{BALB} / \mathrm{c}$ mice at four time points in triplicate after i.v. administration of $3.0 \pm 1.2 \mathrm{MBq}$ (specific activity $12-16$ $\mathrm{GBq} / \mu \mathrm{mol}$ ) (Figure 3). For in vivo PET imaging, $\left[{ }^{18} \mathrm{~F}\right] \mathbf{1}$

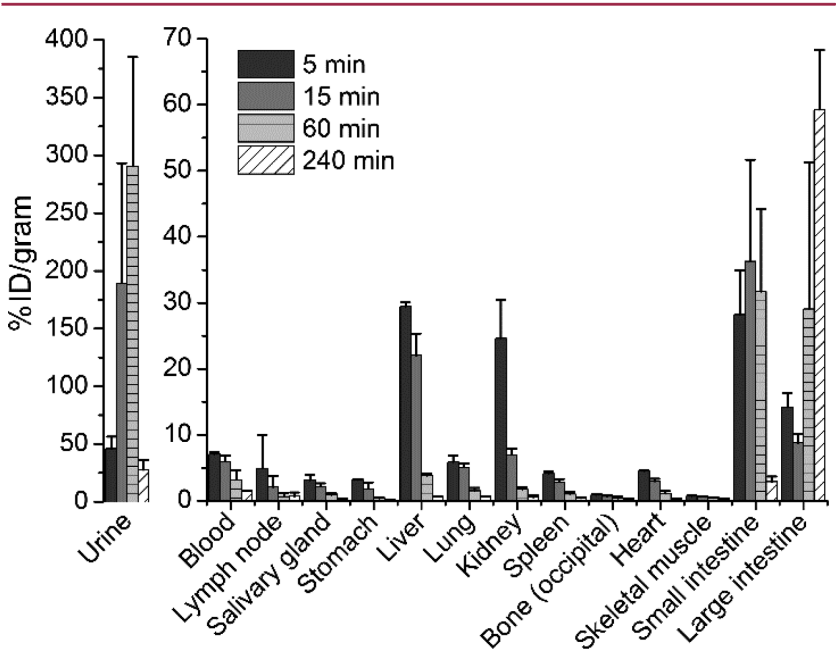

Figure 3. Biodistribution of $\left[{ }^{18} \mathrm{~F}\right] \mathbf{1}$ in $\mathrm{BALB} / \mathrm{c}$ male mice at four time points after i.v. administration of $3.0 \pm 1.2 \mathrm{MBq}$ (specific activity 12$16 \mathrm{GBq} / \mu \mathrm{mol})$. Values represent mean \pm s.d. $(n=3)$.

distribution was recorded with Mediso nanoScan PET-CT for $60 \mathrm{~min}$ in three anesthetized $\left(0.5 \mathrm{~L} / \mathrm{min} \mathrm{O}_{2}, 2 \%\right.$ isoflurane $)$ male BALB/c mice after injection of $5.2 \pm 0.6 \mathrm{MBq}$ (specific activity $302-809 \mathrm{GBq} / \mu \mathrm{mol}$ ) (Figure 4 ). $\left[{ }^{18} \mathrm{~F}\right] 1$ showed only a

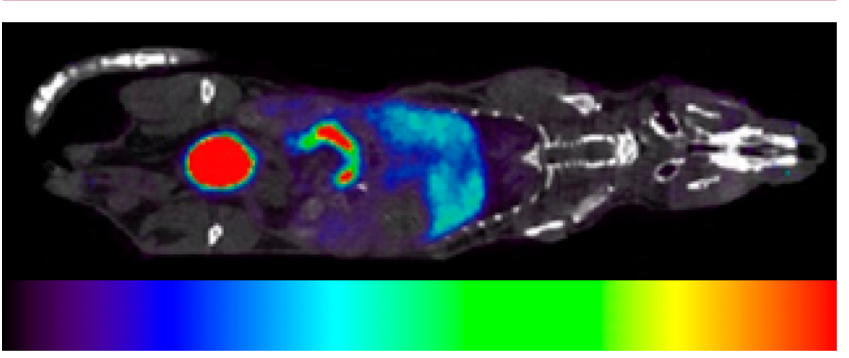

Figure 4. Representative summed (40-60 min) PET-CT image of the biodistribution of radioactivity.

low level of in vivo defluorination; only $1 \%$ of injected dose/ gram was observed in bone after $60 \mathrm{~min}$. The tracer showed fast elimination into urine, accompanied by a lower level of excretion via the hepatobiliary route. Moderate accumulation in liver was observed at the early time points, but it was quickly washed out within the first hour. This confirms our hypothesis that the addition of the $\left[{ }^{18} \mathrm{~F}\right] 6$ moiety to the tetrazine reduces the lipophilicity and promotes desired pharmacokinetics for in vivo bioorthogonal reactions. Plasma radiometabolite analysis revealed that at least $99 \%$ of $\left[{ }^{18} \mathrm{~F}\right] \mathbf{1}$ was intact after $15 \mathrm{~min}$.

The observed quick wash out from the liver differs significantly from the previously reported strong uniform liver accumulation of the actively transported and phosphorylated $\left[{ }^{18} \mathrm{~F}\right]-2-\mathrm{DFR}{ }^{35}$ This indicates that, due to the fluorinated 5position, neither $\left[{ }^{18} \mathrm{~F}\right] \mathbf{1}$ nor its radioactive metabolites are accumulated to the hepatocytes as a result of ribokinase activity. However, contribution of the $\left[{ }^{18} \mathrm{~F}\right] 6$ moiety on the observed intestinal radioactivity still needs further investigation. Although, a more detailed analysis of the observed high intestinal accumulation revealed that it is mainly due to radioactivity of the content: $11.5 \pm 9.0 \% \mathrm{ID} /$ gram (empty 
ileum, $n=3$ ) versus $66.7 \pm 17.4 \% \mathrm{ID} /$ gram (content of ileum, $n$ $=3$ ), and thus originating mainly from the hepatobiliary excretion. During preparation of our manuscript a similarly prepared $\left[{ }^{18} \mathrm{~F}\right]$ FDG-tetrazine was reported and used for the in vitro labeling of antibody fragments. ${ }^{25}$ Similarly to $\left[{ }^{18} \mathrm{~F}\right]-2$ DFR, $\left[{ }^{18} \mathrm{~F}\right] \mathrm{FDG}$ is ${ }^{18} \mathrm{~F}$-fluorinated on its 2 -position, and it is actively transported and phosphorylated in metabolically active cells. Increased heart, liver, and intestinal accumulation has been previously reported for small $\left[{ }^{18} \mathrm{~F}\right]$ FDG-labeled biomolecules such as peptides and oligonucleotides either due to their active transportation by organ specific glucose transporters or due to the metabolic cleavage of $\left[{ }^{18} \mathrm{~F}\right] \mathrm{FDG} .{ }^{37}$ Unfortunately, the biodistribution of the $\left[{ }^{18} \mathrm{~F}\right]$ FDG-tetrazine alone was not reported hampering the comparison of the results to ours, and leaving the possible influence of the selection of the carbohydrate and the labeling position on the pharmacokinetics of glycosylated ${ }^{18} \mathrm{~F}$-tetrazines in vivo open for further investigation.

In summary, a new glycosylated tetrazine derivative, $\left[{ }^{18} \mathrm{~F}\right] \mathbf{1}$, was successfully synthesized under mild reaction conditions with high radiochemical yield, purity, and specific activity. The new tetrazine analogue showed fast reaction kinetics toward the most commonly used dienophiles in inverse electron-demand Diels-Alder cycloaddition and optimal stability under physiological conditions in vitro. In healthy mice, $\left[{ }^{18} \mathrm{~F}\right] 1$ exhibited sufficient blood circulation time and favorable elimination characteristics considering its intended use as a biorthogonal reagent in vivo. In conclusion, these results warrant the use of $\left[{ }^{18} \mathrm{~F}\right] \mathbf{1}$ for in vivo bioorthogonal chemistry. Performance of $\left[{ }^{18} \mathrm{~F}\right] \mathbf{1}$ in pretargeted PET imaging is currently under investigation.

\section{ASSOCIATED CONTENT}

\section{S Supporting Information}

The Supporting Information is available free of charge on the ACS Publications website at DOI: 10.1021/acsmedchemlett.5b00330.

Animal experiment details. Experimental details for the synthesis of compounds $\left[{ }^{18} \mathrm{~F}\right] \mathbf{1}, \mathbf{1}, 4,5$, and $\left[{ }^{18} \mathrm{~F}\right] 6$. ESIMS and HPLC results for the conjugation products of $\mathbf{1}$ with 7, 8, and 9. Lipophilicity measurement, in vitro study, and reaction kinetic measurement details. NMR spectra of 1 and 4 (PDF)

\section{AUTHOR INFORMATION}

\section{Corresponding Author}

*Phone: +358 294150124. E-mail: anu.airaksinen@helsinki.fi.

\section{Author Contributions}

All authors have given approval to the final version of the manuscript.

\section{Funding}

This work was supported by the Academy of Finland (272908, 278056), Doctoral Program in Chemistry and Molecular Science (CHEMS), and ZonMW grant for the Mediso nanoScan PET-CT.

\section{Notes}

The authors declare no competing financial interest.

\section{ACKNOWLEDGMENTS}

The authors thank Dr. Sami Heikkinen for the use of NMR, and Dr. Petri Heinonen for the use of MS. The authors greatly acknowledge Inge de Greeuw, Remco van der Kris, Mariska Verlaan, and Ricardo Vos for biotechnical assistance during PET scans. The help of Berend van der Wildt is greatly appreciated.

\section{ABBREVIATIONS}

CCO, cis-cyclooctene; DIEA, N,N-diisopropylethylamine; DMF, dimethylformamide; HATU, 1-[Bis(dimethylamino)methylene]-1H-1,2,3-triazolo[4,5-b] pyridinium 3-oxid hexafluorophosphate; HPLC, high-performance liquid chromatography; IEDDA, inverse electron-demand Diels-Alder cycloaddition; PET, positron emission tomography; PET-CT, positron emission tomography-computed tomography; RT, room temperature; TCO, trans-cyclooctene

\section{REFERENCES}

(1) Hargreaves, R. J. The role of molecular imaging in drug discovery and development. Clin. Pharmacol. Ther. 2008, 83, 349-53.

(2) Matthews, P. M.; Rabiner, E. A.; Passchier, J.; Gunn, R. N. Positron emission tomography molecular imaging for drug development. Br. J. Clin. Pharmacol. 2012, 73, 175-86.

(3) Bergström, M.; Grahnen, A.; Långström, B. Positron emission tomography microdosing: a new concept with application in tracer and early clinical drug development. Eur. J. Clin. Pharmacol. 2003, 59, $357-66$.

(4) Le Bars, D. Fluorine-18 and medical imaging: Radiopharmaceuticals for positron emission tomography. J. Fluorine Chem. 2006, 127, $1488-1493$

(5) Jacobson, O.; Kiesewetter, D. O.; Chen, X. Fluorine-18 radiochemistry, labeling strategies and synthetic routes. Bioconjugate Chem. 2015, 26, 1-18.

(6) Sletten, E. M.; Bertozzi, C. R. Bioorthogonal chemistry: fishing for selectivity in a sea of functionality. Angew. Chem., Int. Ed. 2009, 48, 6974-98.

(7) Sletten, E. M.; Bertozzi, C. R. From Mechanism to Mouse: A Tale of Two Bioorthogonal Reactions. Acc. Chem. Res. 2011, 44, 666676.

(8) Keliher, E. J.; Reiner, T.; Turetsky, A.; Hilderbrand, S. A.; Weissleder, R. High-yielding, two-step 18F labeling strategy for 18FPARP1 inhibitors. ChemMedChem 2011, 6, 424-7.

(9) Li, Z.; Cai, H.; Hassink, M.; Blackman, M. L.; Brown, R. C.; Conti, P. S.; Fox, J. M. Tetrazine-trans-cyclooctene ligation for the rapid construction of $18 \mathrm{~F}$ labeled probes. Chem. Commun. (Cambridge, U. K.) 2010, 46, 8043-5.

(10) Devaraj, N. K.; Upadhyay, R.; Haun, J. B.; Hilderbrand, S. A.; Weissleder, R. Fast and sensitive pretargeted labeling of cancer cells through a tetrazine/trans-cyclooctene cycloaddition. Angew. Chem., Int. Ed. 2009, 48, 7013-6.

(11) Devaraj, N. K.; Hilderbrand, S.; Upadhyay, R.; Mazitschek, R.; Weissleder, R. Bioorthogonal turn-on probes for imaging small molecules inside living cells. Angew. Chem., Int. Ed. 2010, 49, 2869-72.

(12) Rossin, R.; Renart Verkerk, P.; vanden Bosch, S. M.; Vulders, R. C. M.; Verel, I.; Lub, J.; Robillard, M. S. In Vivo Chemistry for Pretargeted Tumor Imaging in Live Mice. Angew. Chem., Int. Ed. 2010, 49, 3375-3378.

(13) Lewis, M. R.; Wang, M.; Axworthy, D. B.; Theodore, L. J.; Mallet, R. W.; Fritzberg, A. R.; Welch, M. J.; Anderson, C. J. In vivo evaluation of pretargeted $64 \mathrm{Cu}$ for tumor imaging and therapy. J. Nucl. Med. 2003, 44, 1284-1292.

(14) Zeglis, B. M.; Sevak, K. K.; Reiner, T.; Mohindra, P.; Carlin, S. D.; Zanzonico, P.; Weissleder, R.; Lewis, J. S. A pretargeted PET imaging strategy based on bioorthogonal Diels-Alder click chemistry. J. Nucl. Med. 2013, 54, 1389-96.

(15) Blackman, M. L.; Royzen, M.; Fox, J. M. Tetrazine Ligation: Fast Bioconjugation Based on Inverse-Electron-Demand Diels-Alder Reactivity. J. Am. Chem. Soc. 2008, 130, 13518-13519. 
(16) Devaraj, N. K.; Weissleder, R.; Hilderbrand, S. A. TetrazineBased Cycloadditions: Application to Pretargeted Live Cell Imaging. Bioconjugate Chem. 2008, 19, 2297-2299.

(17) Carroll, L.; Evans, H. L.; Aboagye, E. O.; Spivey, A. C. Bioorthogonal chemistry for pre-targeted molecular imaging-progress and prospects. Org. Biomol. Chem. 2013, 11, 5772-81.

(18) Rossin, R; Verkerk, P. R.; van den Bosch, S. M.; Vulders, R. C.; Verel, I.; Lub, J.; Robillard, M. S. In vivo chemistry for pretargeted tumor imaging in live mice. Angew. Chem., Int. Ed. 2010, 49, 3375-8.

(19) Herth, M. M.; Andersen, V. L.; Lehel, S.; Madsen, J.; Knudsen, G. M.; Kristensen, J. L. Development of a (11)C-labeled tetrazine for rapid tetrazine-trans-cyclooctene ligation. Chem. Commun. (Cambridge, U. K.) 2013, 49, 3805-7.

(20) Rossin, R.; van den Bosch, S. M.; Ten Hoeve, W.; Carvelli, M.; Versteegen, R. M.; Lub, J.; Robillard, M. S. Highly reactive transcyclooctene tags with improved stability for Diels-Alder chemistry in living systems. Bioconjugate Chem. 2013, 24, 1210-7.

(21) Seckute, J.; Devaraj, N. K. Expanding room for tetrazine ligations in the in vivo chemistry toolbox. Curr. Opin. Chem. Biol. 2013, 17, 761-7.

(22) Knight, J. C.; Richter, S.; Wuest, M.; Way, J. D.; Wuest, F. Synthesis and evaluation of an 18F-labelled norbornene derivative for copper-free click chemistry reactions. Org. Biomol. Chem. 2013, 11, 3817-25.

(23) Denk, C.; Svatunek, D.; Filip, T.; Wanek, T.; Lumpi, D.; Frohlich, J.; Kuntner, C.; Mikula, H. Development of a (18) F-labeled tetrazine with favorable pharmacokinetics for bioorthogonal PET imaging. Angew. Chem., Int. Ed. 2014, 53, 9655-9.

(24) Zhu, J.; Li, S.; Wangler, C.; Wangler, B.; Bruce Lennox, R.; Schirrmacher, R. Synthesis of 3-chloro-6-((4-(di-tert-butyl[18F]fluorosilyl)-benzyl)oxy)-1,2,4,5-tetrazine ([18F]SiFA-OTz) for rapid tetrazine-based 18F-radiolabeling. Chem. Commun. (Cambridge, U. K.) 2015, 51, 12415-12418.

(25) Rashidian, M.; Keliher, E. J.; Dougan, M.; Juras, P. K.; Cavallari, M.; Wojtkiewicz, G. R.; Jacobsen, J. T.; Edens, J. G.; Tas, J. M. J.; Victora, G.; Weissleder, R.; Ploegh, H. Use of 18F-2-Fluorodeoxyglucose to Label Antibody Fragments for Immuno-Positron Emission Tomography of Pancreatic Cancer. ACS Cent. Sci. 2015, 1, 142-147. (26) Li, X.-G.; Dall'Angelo, S.; Schweiger, L. F.; Zanda, M.; O’Hagan, D. [18F]-5-Fluoro-5-deoxyribose, an efficient peptide bioconjugation ligand for positron emission tomography (PET) imaging. Chem. Commun. (Cambridge, U. K.) 2012, 48, 5247-9.

(27) Li, X.-G.; Autio, A.; Ahtinen, H.; Helariutta, K.; Liljenback, H.; Jalkanen, S.; Roivainen, A.; Airaksinen, A. J. Translating the concept of peptide labeling with 5-deoxy-5-[18F]fluororibose into preclinical practice: 18F-labeling of Siglec-9 peptide for PET imaging of inflammation. Chem. Commun. (Cambridge, U. K.) 2013, 49, 3682-4.

(28) Hill, A. P.; Young, R. J. Getting physical in drug discovery: a contemporary perspective on solubility and hydrophobicity. Drug Discovery Today 2010, 15, 648-655.

(29) Trainor, G. L. The importance of plasma protein binding in drug discovery. Expert Opin. Drug Discovery 2007, 2, 51-64.

(30) Fauber, B. P.; René, O.; de Leon Boenig, G.; Burton, B.; Deng, Y.; Eidenschenk, C.; Everett, C.; Gobbi, A.; Hymowitz, S. G.; Johnson, A. R.; La, H.; Liimatta, M.; Lockey, P.; Norman, M.; Ouyang, W.; Wang, W.; Wong, H. Reduction in lipophilicity improved the solubility, plasma-protein binding, and permeability of tertiary sulfonamide RORc inverse agonists. Bioorg. Med. Chem. Lett. 2014, 24, 3891-3897.

(31) Arnott, J. A. The influence of lipophilicity in drug discovery and design. Expert Opin. Drug Discovery 2012, 7, 863-75.

(32) Decristoforo, C.; Mather, S. J. 99m-Technetium-labelled peptide-HYNIC conjugates: effects of lipophilicity and stability on biodistribution. Nucl. Med. Biol. 1999, 26, 389-396.

(33) Lin, K.-S.; Luu, A.; Baidoo, K. E.; Hashemzadeh-Gargari, H.; Chen, M.-K.; Brenneman, K.; Pili, R.; Pomper, M.; Carducci, M. A.; Wagner, H. N. A New High Affinity Technetium-99m-Bombesin Analogue with Low Abdominal Accumulation. Bioconjugate Chem. 2005, 16, 43-50.
(34) Knight, J. C.; Cornelissen, B. Bioorthogonal chemistry: implications for pretargeted nuclear (PET/SPECT) imaging and therapy. Am. J. Nucl. Med. Mol. Imaging 2014, 4, 96-113.

(35) Evdokimov, N. M.; Clark, P. M.; Flores, G.; Chai, T.; Faull, K. F.; Phelps, M. E.; Witte, O. N.; Jung, M. E. Development of 2-Deoxy2 -[18F]fluororibose for Positron Emission Tomography Imaging Liver Function in Vivo. J. Med. Chem. 2015, 58, 5538-5547.

(36) Wijnen, J. W.; Zavarise, S.; Engberts, J. B. F. N.; Charton, M. Substituent Effects on an Inverse Electron Demand Hetero DielsAlder Reaction in Aqueous Solution and Organic Solvents: Cycloaddition of Substituted Styrenes to Di(2-pyridyl)-1,2,4,5-tetrazine. J. Org. Chem. 1996, 61, 2001-2005.

(37) Hultsch, C.; Schottelius, M.; Auernheimer, J.; Alke, A.; Wester, H.-J. 18F-Fluoroglucosylation of peptides, exemplified on cyclo(RGDfK). Eur. J. Nucl. Med. Mol. Imaging 2009, 36, 1469-1474. 